\author{
ANNALS OF “DUNAREA DE JOS” UNIVERSITY OF GALATI \\ MATHEMATICS, PHYSICS, THEORETICAL MECHANICS \\ FASCICLE II, YEAR VIII (XXXIX) 2019, No. 1
}

Article DOI: $\underline{\text { https://doi.org/10.35219/ann-ugal-math-phys-mec.2019.1.16 }}$

\title{
LASER-INDUCED PERIODIC SURFACE NANOSTRUCTURES FORMATION AND MODIFICATION IN POLYETHYLENE TEREPHTHALATE (PET)
}

\author{
Radu Costin Pană ${ }^{1}$ \\ ${ }^{1}$ University of Bucharest, Faculty of Physics, Bucharest, Romania, email: radu.pana@ gmail.com
}

\begin{abstract}
Polyethylene terephthalate (PET) is a thermoplastic polymer used in a large variety of industries as clothing, packaging and aeronautics among many others. Laser-inducted periodic surface nanostructures presents great potential as long as the modifications of materials are delivered in a controlled manner. However the formation of nanostructures having different morphological properties presents itself a difficult errand. The purpose of this paper is to present how the surface structure of Polyethylene terephthalate (PET) changes at nanoscale levels after irradiation with laser beams of spatially variant polarization. Utilizations of such nanostructures can be seen in a multitude of applications varying from electronics and textiles to aeronautical and military implementations. For the creation of the new nanostructures has been used an YAG solid state laser (Spectra Physics, Quanta Ray) with a pulse duration of $4 \mathrm{~ns}$ and a repetition rate of $10 \mathrm{~Hz}$ for different spatial periods and at different inclination angles, an Atomic Force Microscope and a Scanning Electron Microscope in order to analyze the newly created nanostructures. Such Polyethylene terephthalate (PET) modified structures have been used as well in combination with fiber glass compounds in order to produce highly resistant engineering resins to heat and impact. The formation of specific controllable surface nanostructures given different functionalities are of major interest as a technique to improve or even produce completely new specific oriented properties. This study reveals how the structure of polyethylene terephthalate surface changes after laser-induced treatment.
\end{abstract}

Keywords: polyethylene terephthalate, laser-induced treatment, polymer surface modifications.

\section{INTRODUCTION}

The purpose of this paper is to present how the surface structure of Polyethylene terephthalate (PET) changes at nanoscale levels after irradiation with laser beams of spatially variant polarization. Utilizations of such nanostructures can be seen in a multitude of applications varying from electronics and textiles to aeronautical and military implementations [1].

Such Polyethylene terephthalate (PET) modified structures have been used as well in combination with fiber glass compounds in order to produce highly resistant engineering resins to heat and impact [2].

Laser induced periodic surface structuring (LIPSS) is a special Laser-inducted technique so that the surface functionalization can be modified and thus applied in various and diverse fields that require all different kinds of material surfaces, including metals, semiconductors, and dielectrics [3]. 
The formation of specific controllable surface nanostructures given different functionalities are of major interest as a technique to improve or even produce completely new specific oriented properties. This study reveals how the structure of polyethylene terephthalate surface changes after laser-induced treatment [4].

\section{NANOSTRUCTURE FORMATION AND INVESTIGATION}

For the creation of the new nanostructures has been used an YAG solid state laser (Spectra Physics, Quanta Ray) with a pulse duration of $4 \mathrm{~ns}$ and a repetition rate of $10 \mathrm{~Hz}$ for different spatial periods and at the inclination angles of $30^{\circ}$ and $45^{\circ}$.

A scanning electron microscopy (SEM), an Atomic Force Microscope (AFM) and a confocal microscope have been used in order to study the newly created nanostructure at the surface of the nanocomposite.

The cross-section of the newly fabricated nanostructure is measured with the help of a confocal microscope and it is shown in the Figure 1a. It can be seen, that the resultant structure contains periodic line-like grooves formed of inclined planes.

In the Figure $1 \mathrm{~b}$. and Figure 1c. can be seen images of the formed structure from the magnified SEM image and the formation of the LIPSS oriented perpendicularly to the direction of the structure can be observed on top of those planes.

As well the LIPSS depth was investigated with an atomic force microscopy (AFM) and the analysis concluded that the irradiated area contains nanowaves with extremely similar heights of an average depth of around $\sim 150 \mathrm{~nm}$. Figure 2a. shows the surface after irradiation under an angle of incidence of $30^{\circ}$ and Figure $2 \mathrm{~b}$. after irradiation under an angle of incidence of $45^{\circ}$ respectively.
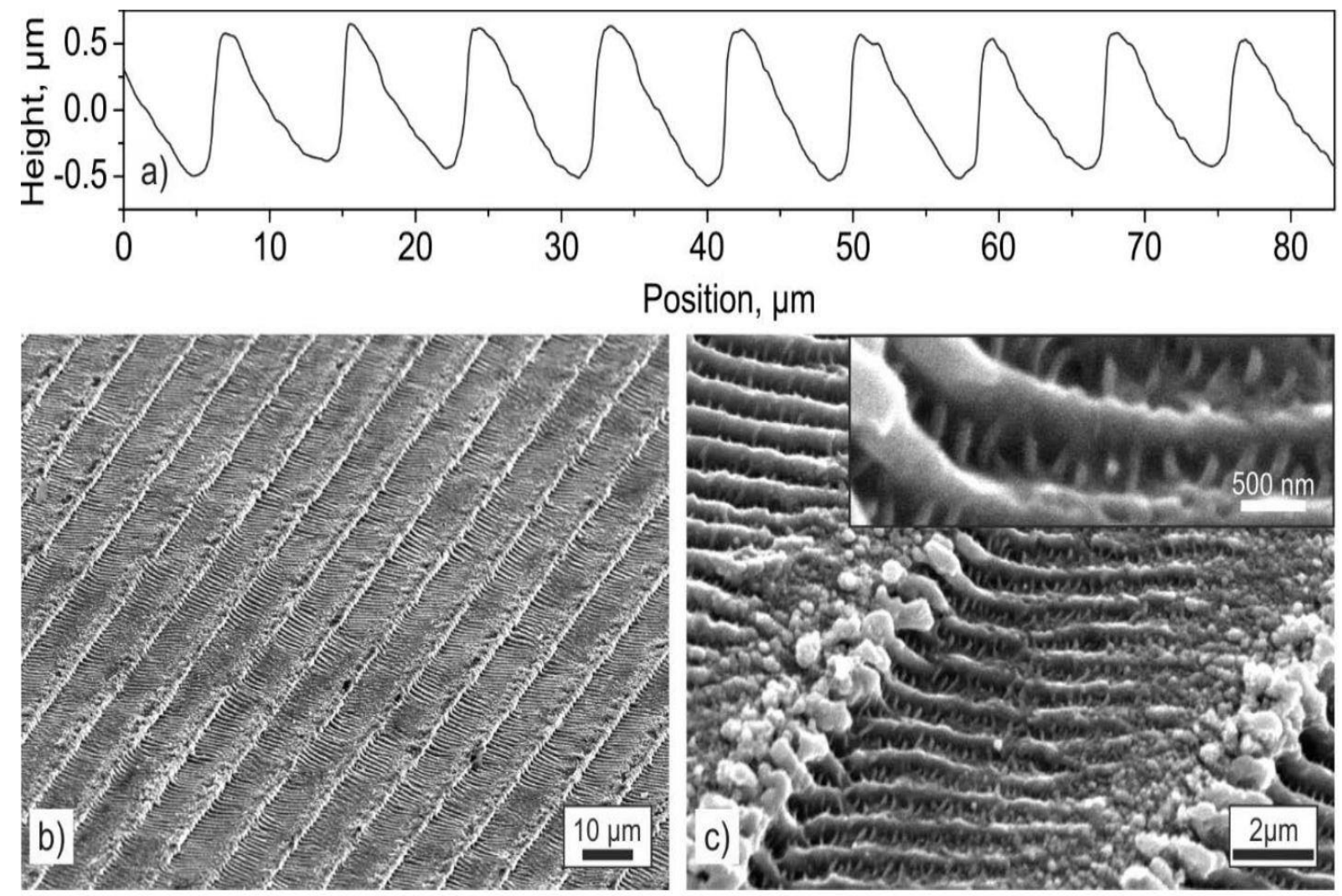

Fig. 1.Profile of the structure formed on PET surface and the Scanning Electron Microscope images of the same surface created with laser pulses under the angle of $45^{\circ}$ at various investigation distances 

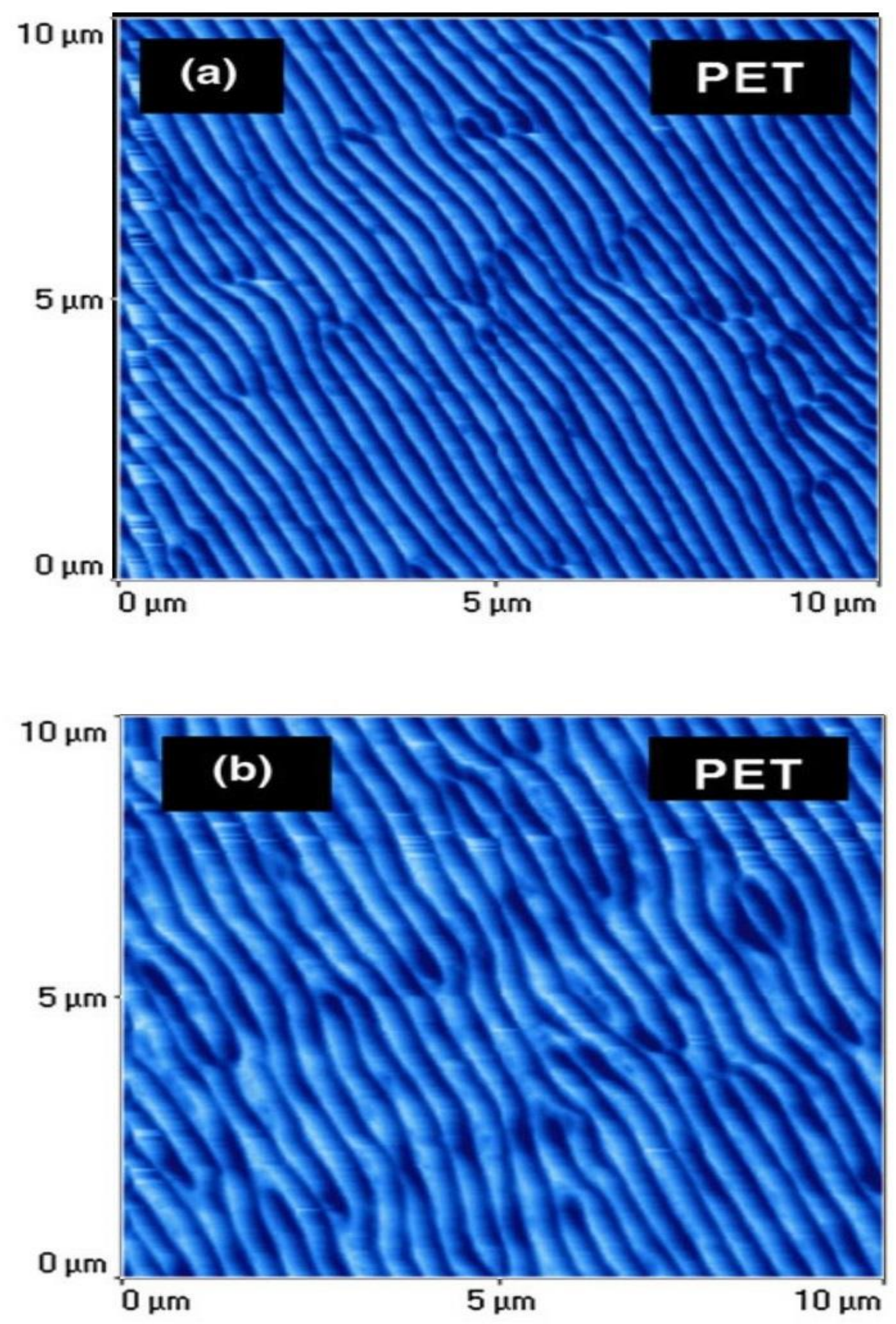

Fig. 2. Atomic Force Microscope images of Laser-inducted periodic surface structures on PET after irradiation with laser pulses under an angles of incidence of $30^{\circ}(a)$ and $45^{\circ}(b)$ 


\section{CONCLUSIONS}

Laser induced periodic surface structures (LIPSS) created by YAG laser can be used for nanopatterning of relative large areas on synthetic polymers commonly used as cell culture substrates.

Regular spikes with a groove amplitude of $20 \mathrm{~nm}$ (period of $266 \mathrm{~nm}$ ) were observed by SEM and AFM after laser irradiation at a fluency below the ablation threshold with 1000 shots and $\mathrm{f}=10 \mathrm{~Hz}$. We see a large potential for this type of structures, especially in the field of tissue engineering and biosensors. In the latter case we think especially of surface resonance sensors directly integrated into biotechnological devices.

\section{References}

1. Zorba V., Persano L., Pisignano D., Athanassiou A., Stratakis E., Cingolani R., Tzanetakis P., Fotakis C., Making silicon hydrophobic: wettability control by two-lengthscale simultaneous patterning with femtosecond laser irradiation, Nanotechnology 17(13), pp. 3234-3329, 2006.

2. Siegel J., Heitz J., Reznickova A., Svorcik V., Preparation and characterization of fully separated gold nanowire arrays, Appl. Surf. Sci., 264, pp. 443-447, 2013.

3. Burakov I. M., Bulgakova N. M., Stoian R., Rosenfeld A., Hertel I. V., Theoretical investigations of material modification using temporally shaped femtosecond laser pulses, Appl. Phys. A: Mater. Sci., 81(8), pp. 1639-1645, 2005.

4. Nolte S., Momma C., Kamlage G., Ostendorf A., Fallnich C., von Alvensleben F., Welling H., Polarization effects in ultrashort-pulse laser drilling, Appl. Phys. A: Mater. Sci., 68(5), pp. 563-567, 1999. 